\title{
Motility and fitness of microorganisms in dynamic aquatic ecosystems: a simulation study
}

\author{
A.I. Klimenko \\ Kurchatov Genomics Center, \\ ICG SB RAS \\ Novosibirsk, Russia \\ klimenko@bionet.nsc.ru
}

\author{
Yu.G. Matushkin \\ ICG SB RAS \\ Novosibirsk, Russia
}

\author{
S.A. Lashin \\ Kurchatov Genomics Center, \\ ICG SB RAS, Novosibirsk, Russia \\ Novosibirsk State University, \\ Novosibirsk, Russia
}

\begin{abstract}
Motility is one of the key factors of adaptation in scarce marine environments inhabited by bacteria. This simulation study addresses the question of how an ability for adaptive migrations influences the evolutionary success of a population in various conditions. We examined the model of competition of motile and sessile populations of microorganisms in a confined aquatic environment supplied with a periodic batch nutrient source and assessed the fitness of both. We studied the effect of such factors as nutrient concentration in a batch, batch period, mortality term and penalty for migration and determined the conditions favouring "nomad" strategy of motile population and those favouring "settler" strategy of sessile one.
\end{abstract}

Key words - motility; marine bacteria; agent-based modelling; ecological modelling

\section{Motivation and Aim}

In contrast to well-studied symbiotic gut microbiota living in abundant conditions, marine bacteria live in the world of scarce and ephemeral nutrition sources. Therefore, motility becomes one of the key factors of adaptation in these conditions. However, the advantage granted by motility comes with its cost and current estimates show that energy expenditure of various marine bacteria ranges from $2 \%$ to $50 \%$ of their total energy budgets [1] with different species varying in motility. While the processes of adaptation mediated by chemotaxis are relatively well-studied at the micro-scale level, there is a lack of understanding of how an ability for adaptive migrations influences the evolutionary success of a population.

\section{Methods and Algorithms}

We have used the Haploid Evolutionary Constructor 3D (HEC 3D) [2] software complex to build the model of competition of motile and sessile populations of microorganisms in a confined aquatic environment supplied with a periodic batch nutrient source. The HEC 3D allows creating multilayer ecological models of microbial populations inhabiting spatially structured environments. To describe a motile population capable of adaptive migrations via chemotaxis more adequately, the basic functionality of HEC 3D was extended to take into account the variability in migration traits such as energetic cost of migration.

\section{Results}

To estimate what conditions allow the motile population to make use of its advantage and dominate over the sessile one, we have investigated a model of a community consisting of two microbial populations in different model scenarios varying such parameters as nutrient concentration in a batch, batch period, mortality term and penalty for migration. It turned out that more dynamic and scarce environments favour dominance of motile populations whereas rich and stagnant environments tend to promote the dominance of sessile microorganisms. Moreover, dense-dependent quadratic mortality is more detrimental for motile population than linear one. We have found out that there is a turning point in the migration penalty value determining whether "nomad" strategy of motile population is adaptive or not. However, this value heavily depends on such conditions as nutrient availability. We have also shown that even without penalties for migration sessile population following the "settler" strategy can achieve dominant position in the ecosystem via decreasing local nutrient availability in the nutrient source's vicinity.

\section{Conclusion}

Thus, motile populations relying on local optimizing strategy tend to follow benign conditions and fail enduring stress associated with crossing the valleys of suboptimal nutrient availability. Though providing more adequate description, taking into account energetic cost of migration does not change the major trends in the competition between two types of microorganisms relying on different strategies in terms of motility.

\section{ACKNOWLEDGMENT}

This study was funded by the Budget Project No. 03242019-0040-C-01.

\section{REFERENCES}

[1] R. Stocker and J. R. Seymour, "Ecology and physics of bacterial chemotaxis in the ocean.," Microbiol. Mol. Biol. Rev., vol. 76, no. 4, pp. 792-812, 2012.

[2] A. I. Klimenko, Y. G. Matushkin, N. A. Kolchanov, and S. A. Lashin, "Modeling evolution of spatially distributed bacterial communities : a simulation with the haploid evolutionary constructor," BMC Evol. Biol., vol. 15, no. Suppl 1, p. S3, 2015 . 